\title{
Helping Hands: Designing Video Games with Interpersonal Touch Interaction
}

\author{
Cody Watts ${ }^{1,2}$, Ehud Sharlin ${ }^{1}$, and Peter Woytiuk ${ }^{2}$ \\ ${ }^{1} 1$ University of Calgary, 2500 University Drive NW, Calgary, Alberta, Canada \\ ${ }^{2}$ BioWare Corp., Edmonton, Alberta, Canada \\ \{watt.sc, ehud\}@cpsc.ucalgary.ca, woytiuk@bioware.com
}

\begin{abstract}
Increasingly, the movements of players' physical bodies are being used as a method of controlling and playing video games. This trend is evidenced by the recent development of interpersonal touch-based games; multiplayer games which players control by physically touching their partners. Although a small number of interpersonal touch-based games have recently been designed, the best practices for creating video games based on this unconventional interaction technique remain poorly explored and understood. In this paper, we provide an overview of interpersonal touch interaction in video games and present a set of design heuristics for the effective use of interpersonal touch interaction in video games. We then use these heuristics to analyze three current interpersonal touch-based games in order to show how these heuristics reflect on the current state of the art. Finally, we present our vision for the future of this interaction modality in video games.
\end{abstract}

Keywords: Games, interpersonal, touch, interface, design, heuristics.

\section{Introduction}

Within the last decade, the way we play video games has changed. The phenomenal success of games like Dance Dance Revolution, Guitar Hero and Rock Band has ushered in a new era of physically-active gaming. To see the popularity of these physically-active games, one need look no farther than Nintendo's home fitness game, Wii Fit which (as of this writing) has sold 22.6 million copies, making it the fifth-bestselling video game ever produced. The success of these games and of many others like them has forced both players and game designers to broaden their understanding of what it means to play video games. Video gaming is no longer just an activity which involves passively staring at a computer monitor - it can also be an active experience, one which involves the player's entire body.

One of the recent advancements in the field of physically-active gameplay is the advent of video games based on interpersonal touch. These are multiplayer video games in which the system can sense when two or more players are physically touching, and which makes the act of touch part of the game. The distinguishing characteristic of interpersonal touch-based games is not just that the players' physical actions have a direct impact on the game they are playing, but that these actions arise through their interactions with their fellow players. In this regard, games based on 
interpersonal touch provide an inherently social experience, since their gameplay necessitates interaction between players.

We are aware of only four video games which have made use of interpersonal touch in their gameplay mechanics: "Get Lucky" Charms (also known as Intimate Controllers) by Chowdhury [3], Freqtric Game by Baba [1], and Matchmaker and Prism Squad: GO!, both designed by our group [11, 10, respectively]. As these four games form the basis of our discussion in this paper, we shall examine each of them in detail later on. But first, we turn our attention to a more fundamental question: How does interpersonal touch fit into the broader practice of video game design?

\section{Interpersonal Touch in Video Games}

If we accept that the purpose of a game is to provide amusement to its players, then any effective use of interpersonal touch must somehow contribute to this overarching goal. We believe that interpersonal touch can best contribute to players' enjoyment by promoting player-to-player socialization. The value of socializing in multiplayer video games should not be underestimated; several studies have shown that the social atmosphere created by video games can be just as important to players' enjoyment as the game they are playing. In "Why We Play Games: Four Keys to More Emotion Without Story", Lazarro examined thirty volunteers while they played their favorite video games in an attempt to understand what makes games fun [8]. She encapsulated her findings in the form of four "keys" - aspects of gameplay which players enjoyed. Lazzaro's fourth key, which she called "The People Factor," described the rewarding interactions which come from socializing with other players. Lazarro observed that "players in groups emote more frequently and with more intensity than those who play on their own. Group play adds new behaviors, rituals, and emotions that make games more exciting." In fact, her findings indicate that the act of socializing may often take precedence over the game itself - in many cases, players would "play games they don't like [just] so they can spend time with their friends." [8]

Voida et al. reported similar findings in their study of collocated gaming groups [9]. In their own words: "The primary motivation for group console gaming was not the games themselves, but the social interactions afforded by the collocated gameplay. The most important part of group console gaming was, very simply, 'the sociability of it"'. In fact, for many participants in Voida's study, socialization was not just the primary motivation for playing but the only reason: many of the adult females and all of the elderly participants who were interviewed for the study admitted that they only played console games in groups - never by themselves [9].

We believe that interpersonal touch provides opportunities for socialization in a media where social interaction is desirable. Human beings naturally recognize touch as a gesture which signifies a meaningful social connection; when two players touch, it acts as an icebreaker and as an invitation to further social interaction - even if the players themselves don't consciously realize this [4]. By integrating interpersonal touch into the designs of video games, we believe it becomes possible to create more social, more memorable gameplay experiences. 


\section{Video Games Based on Interpersonal Touch}

With our newfound understanding of interpersonal touch and how it shapes players' gameplay experiences, let us now examine how interpersonal touch has been used in practice. We begin our examination of interpersonal touch in games with Intimate Controllers [3] - a project which combined video gaming with the intimate aspects of touch.

\subsection{Intimate Controllers}

Intimate Controllers was an exhibit presented at Unravel, the SIGGRAPH 2007 fashion show. The eponymous intimate controllers were pair of wearable undergarments a woman's bra, and a man's boxer-short - designed to be used as wearable video game controllers. Each controller contained a set of embedded touch sensors which were divided into three "intimacy levels". The more intimate touch-sensors were positioned nearer to the cups of the bra and the buttocks of the shorts, respectively. These controllers were designed to be used by couples as a way of encouraging intimate interaction between the partners as they played. Each player's inputs are located on the opposite player's body - the male player touches his partner's bra, and the female player touches her partner's underpants. Due to the layout of the sensors, this leads to very sexually-explicit positioning when two partners play together: the male stands behind his female partner, cupping her bra, while the female player reaches behind herself to cup the male's buttocks [3].

Accompanying these Intimate Controllers was a video game entitled "Get Lucky" Charms. "Get Lucky" Charms was a timing-based game similar to Dance Dance Revolution in which players had to touch their partner in the appropriate location as symbols corresponding to the various intimacy levels scrolled from the bottom to the top of the screen.

\subsection{Freqtric Game}

Freqtric Game is the unifying label applied to three interpersonal touch-based games developed by Baba et al [1]. Each game in the Freqtric Game series is played using the "Freqtric Game controller device" - a handheld gamepad with a steel plate on the back which enables the system to detect when two players are touching. The three games each explore the use of touch in a different genre of gameplay. Freqtric Shooting is a two-player cooperative top-down shoot-'em-up where players can touch their partner to activate screen-clearing bombs. Defeated players can also rejoin the game by touching their partner 100 times in a row. Freqtric Dance is a Dance Dance Revolution clone which introduces a "touch" symbol to the standard directional arrows. Freqtric Robot Battle is a competitive two-player fighting game which plays out like a robot sumo match; each player's goal is simply to knock their opponent out of the ring. In addition to firing a virtual missile launchers, players can attack their opponent's robot by physically attacking their opponent; slapping the other player will cause their robot to recoil, while grabbing your opponent will allow your robot to drag your opponent's robot around the arena. 


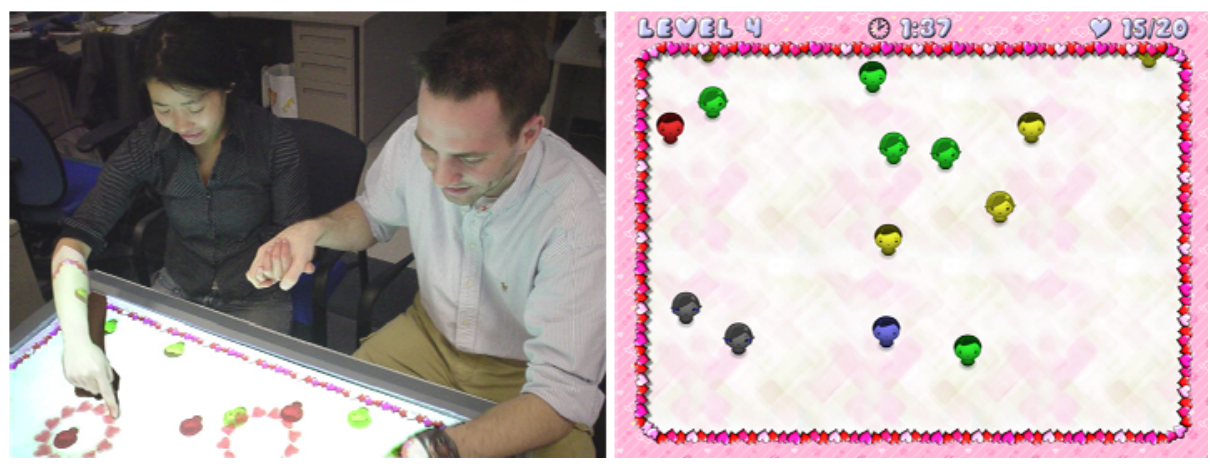

Fig. 1. A couple playing Matchmaker (left). Matchmaker's main game screen (right). [11]

\subsection{Matchmaker}

Matchmaker is a two-player, cooperative tabletop video game which, like Intimate Controllers, examines the role of touch as a symbol of romantic love between couples [11]. But whereas Intimate Controllers put its emphasis on sexual intimacy, Matchmaker focuses on the cute, more innocent aspects of love. [11]

In Matchmaker, players work as a team to create matches between tiny virtual people called "Peeps" (Fig. 1). Players can move Peeps around the tabletop by touching and dragging them with their fingers. When the players select two Peeps and drag them together, a match will be created if the Peeps are the same color. The players' goal is simply to make as many matches as they can. However, there is a catch: If Peeps are not matched up quickly enough they will become "lovelorn". Lovelorn Peeps quickly turn grey, making them ineligible to be matched with other Peeps. Lovelorn Peeps can only be cured through interpersonal touch. If a player holds their partner's hand and touches a lovelorn Peep then the Peep's color will return, allowing it to be matched up once more.

Matchmaker shows how interpersonal touch can serve two functions - it is both a gameplay mechanic and a social dynamic. In gameplay terms, handholding is a cooperative mechanic which encourages players to work together in their pursuit of a shared goal. However, it is also a tangible symbol of the game's romantic theme.

\subsection{Prism Squad: GO!}

Whereas the previous two games explored the use of interpersonal touch in romantic contexts, Prism Squad: GO! was our attempt to broaden the appeal of interpersonal touch in games by creating a touch-based game which could be enjoyed by anyone not just romantic couples [10]. Prism Squad: GO! is a science-fiction shoot-'em-up, in which players defend a planet from invading UFOs by using their personal spacecraft to shoot down the incoming UFOs before they can crash into the surface of the planet. The game is played using Nintendo Wiimote controllers and a large-screen display.

Prism Squad: GO! is a game which makes extensive use of color. Both the players and the enemies are color-coded. This is significant because an enemy UFOs can only be destroyed by a player of the same color. And while there are only three players 
(red, yellow and blue), the enemies come in seven distinct colors (red, yellow, blue, orange, green, purple and white.) While the red, yellow and blue UFOs can be destroyed by a single player acting alone, players must use teamwork to defeat the orange, green, purple and white UFOs. This teamwork is manifested through Prism Squad's color-blending mechanic: whenever two players touch, their colors will temporarily be combined, changing the color of each player involved. For example, if the red and blue players touch, their spacecrafts will each turn purple, temporarily allowing them to destroy purple UFOs. If all three players touch, their spacecrafts will turn white. Much of the excitement in Prism Squad: GO! comes from deftly coordinating blends with your teammates to take on the ever-changing onslaught of colorful UFOs.

Although Prism Squad: GO!'s gameplay mechanics were inspired by interpersonal touch, we are currently still developing a working touch-sensor for the game [10]. Therefore, we used a form of "simulated touch" to develop and playtest Prism Squad: GO! We designated the 'A' button on each player's Wiimote as the "touch button" whenever two or more players pressed this button simultaneously, the game client behaved as if those players were touching and blended their colors together. Although this method of simulating touch cannot capture the physical sensation of interpersonal touch, we believe it adequately simulates interpersonal touch's cooperative nature.

We evaluated Prism Squad: GO! in a series of playtest sessions with volunteer participants [9]. Observations of the players' behavior, combined with testimony from the players themselves suggested that Prism Squad's cooperative, team-based gameplay encouraged players to communicate and interact with their teammates as they played. When the game ended, we asked participants to describe how they thought the game would be different if interpersonal touch was used to blend colors instead of simultaneous button-pressing. Although many participants felt that interpersonal touch would further encourage the rewarding inter-player interactions which players already enjoyed, some participants warned that the addition of interpersonal touch could make it awkward to play the game with strangers, or with members of the opposite sex. Some participants also argued that it may be difficult to get close enough to physically touch your partner, especially in situations where a third player is blocking your way.

\section{Heuristics for Designing Games Based on Interpersonal Touch}

Like all interaction techniques, interpersonal touch has its own pitfalls, limitations, and best practices. However, because there are so few video games which have made use of interpersonal touch in their gameplay mechanics, there have been very few opportunities to study this interaction technique in practice. This places game designers who are interested in creating video games based on interpersonal touch at a disadvantage; without a foundation of understanding of how to use interpersonal touch effectively, every designer must - through trial and error - devise their own best practices from scratch. The following heuristics are our attempt to address this situation. Here, we present our guidelines for the effective application of interpersonal touch interfaces to video games. These heuristics come directly from the lessons we learned designing, developing, refining and evaluating Matchmaker and Prism Squad: GO! These heuristics can be used as both a framework to evaluate a video game's use of interpersonal touch (as we will demonstrate in section 5), or as suggested guidelines for the design of future games based on touch. Our heuristics are: 
i. Use Touch to Simplify Collaborative Interactions between Players

ii. Use Touch to Create Challenge

iii. Use Touch to Encourage Socialization between Players

iv. Manage Players' Social Expectations

v. Design for the Physical Limitations of Touch

\section{i. Use Touch to Simplify Collaborative Interactions between Players}

One of the understated advantages of interpersonal touch is that it is a simple and natural way for two or more players to interact with each other. Touch is never something which needs to be taught or explained. It is simple and natural to reach out and touch someone as a method of demonstrating a connection with that person and it is just as simple to withdraw your hand to break such a connection. In any gameplay situation where two or more players are performing some action which affects them jointly, interpersonal touch is a quick and easy way to link these players for the duration of their action.

\section{ii. Use Touch to Create Challenge}

Although interpersonal touch can sometimes streamline interactions between players, touch is not always the fastest or most efficient way to interact - at times, forcing players to interact through touch can also make their gameplay more difficult. Forcing players to move and interact with their partners in the real world takes time, energy and coordination especially when more than two players are involved. Thus, interpersonal touch can be used to make ordinarily simple tasks more challenging - especially in cases where when players must act quickly.

Though this added challenge may seem like something to avoid, consider the purpose of game design: the goal is not to make a game which is easy, but a game which is fun. Oftentimes it is necessary to challenge players in order to maintain their interest and enjoyment - by challenging your players, you encourage them to strategize, to learn and to grow. As games-theorist Ralph Koster wrote: "true fun is the emotional response to learning" [7].

Interpersonal touch challenges players by forcing them to split their awareness between the digital world and the physical one. In a touch-based game, players must dedicate as much attention to their physical setting and the locations of their partners as the action happening onscreen. Requiring players to coordinate their actions in two separate worlds, creates a new gameplay paradigm - a new cognitive environment to explore and ultimately, a new way to have fun.

\section{iii. Use Touch to Encourage Socialization between Players}

Interpersonal touch is an inherently social gesture - at least in the sense that it requires two or more players to occur. An act of interpersonal touch quite literally forms a connection between those involved, and existing research suggests that this connection is as much emotional as it is physical $[2,4,6]$. This socio-emotional connection is of great importance to game design because, for many players, the act of socializing is actually more important than the act of gameplay $[8,9]$. Encouraging players to interact through touch, creates a catalyst for ongoing social interaction. A shared touch between two players tacitly breaks down social barriers and encourages further 
dialogue. For example, in Prism Squad: GO! discussions about how and when to blend colors were common among players [10]. Although these conversations were strictly focused on gameplay, we believe that this ongoing dialog also contributed to a social atmosphere between players. Based on our observations, the more players strategized, the more they talked with their teammates, even during the periods of relaxation between stages [10]. When used effectively, interpersonal touch stimulates social interactions between players, and leads naturally towards the "behaviors, rituals, and emotions that make games more exciting." [8]

\section{iv. Manage Player's Social Expectations}

Players do not approach your game as a blank slate; for better or for worse, each player will come to you with their own ideas about what interpersonal touch means to them. Making an effective use of interpersonal touch requires designers to anticipate these ideas, and design their games accordingly.

For most players, touch represents connection, attachment, intimacy, or even love. In some situations, this can work to a designer's benefit; a romantically-themed game such as "Get Lucky” Charms [3] or Matchmaker [11] can use romantic gestures such as handholding as a way to reinforce its message of love and compassion. However, in other game designs, interpersonal touch may be seen as undesirable or even inappropriate - players may feel uneasy about playing games based on touch if they are paired with members of the same sex or players with whom they are not wellacquainted. Your players' cultural and religious backgrounds will also play a role in determining how they feel about interpersonal touch; acts of touch which are permissible in one culture may be stigmatized in another.

We do not believe that this means that interpersonal touch should be strictly relegated to romantically-themed games. But it does mean that game designers must be mindful about their potential audience, and their attitude towards touch. If your players might be uncomfortable with touching their partners, then it may be worthwhile to encourage less intimate forms of interpersonal touch, such as touching on the shoulder or back.

\section{v. Design for the Physical Limitations of Touch}

As a physical interaction technique, interpersonal touch is subject to physical limitations which constrain the types of interactions that it can realistically support. The most obvious limitation on interpersonal touch is simply that, in order to for two or more players to touch they must be in close physical proximity. That is to say: a game based on interpersonal touch can only be played by two or more players in a collocated group. However, designers must also be careful not to involve too many players, especially if they rely on a single, shared screen; as more and more players become involved, it becomes more and more difficult for two players to touch at any given time simply because of the increasing number of bodies in the way. When creating games that involve more than two players, designers must be careful to avoid creating situations where one player can interrupt another by reaching for a third. This is especially true for tabletop games, where players may need to reach across the table to touch someone on the opposite side. 
Another limitation of interpersonal touch is that it requires at least one hand free. This restriction makes it quite difficult to use interpersonal touch in conjunction with two-handed controllers such as gamepads. This limitation encourages designers to work with "hands-free" interfaces such as tabletop surfaces, or one-handed pointing devices, like the Nintendo Wiimote.

\section{Evaluating the Interpersonal Touch Game Design Heuristics}

Having outlined our five design heuristics, let us now see how they apply to the four interpersonal touch-based video games which we have previously introduced: "Get Lucky" Charms, Freqtric Game, Matchmaker and Prism Squad: GO! By analyzing these games in terms of the heuristics we have presented, we seek to show how our heuristics reflect in practice on the design of interpersonal touch-based games. In the following section, whenever we reflect on a heuristic we will follow it with its heuristic number in parentheses, e.g. $(h-i i)$.

\section{1 "Get Lucky" Charms}

In many ways "Get Lucky" Charms [3] makes very effective use of interpersonal touch. It presents a very powerful narrative about touch's role in love, intimacy and sexuality - everything from the title of the project, to the design of the intimate controllers themselves suggests a playful atmosphere where interpersonal touch is very appropriate $(h-i v)$. The game is clearly designed for couples and it takes advantage of this design choice by using peripherals which force the players to stand with their bodies pressing against each other $(h-v)$. The sensors on the Intimate Controllers are positioned to encourage players to touch their partners' breasts and buttocks, which is designed to evoke feelings of intimacy between the players $(h-i i i)$.

Although it is clear that great care has been spent on the design of "Get Lucky" Charms' interface, we feel that less attention has been spent on the design of the accompanying game. In fact, the game itself has very little substance and seems to exist for no other reason than to get the players touching - the gameplay seems to be nothing more than a series of prompts to touch your partner in a specific location on their body. As a result, the game requires absolutely no collaboration between its players (h-i). In "Get Lucky" Charms each player acts as a dumb receptor for their partner's touch and so players are never actively encouraged to strategize, or to communicate about what they're doing ( $h$-iii). Based on these observations, it seems as though the entire point of "Get Lucky" Charms is simply to put players in a situation where they can touch each other in an intimate way. That is not necessarily a bad thing - but if that is the case, why make a video game of it? We argue that there are more romantic ways to get close to your significant other - ways which do not even require you to get dressed in sensor-equipped undergarments first.

\subsection{Freqtric Game}

If nothing else, Freqtric Game shows how versatile interpersonal touch-based gaming can be; Freqtric Shooting, Freqtric Dance and Freqtric Robot Battle all approach touch from very different perspectives. In Freqtric Shooting, interpersonal touch is 
very much a cooperative action - players must touch to activate life-saving "bombs" $(h-i)$. Since players' supplies of bombs are limited, this encourages players to communicate about when and how to use them $(h$-iii). The mechanic of reviving a downed player through interpersonal touch is somewhat suspect, though; being quickly tapped 100 times in a row by one's partner is more likely to annoy players than anything else (h-iv). Freqtric Dance uses interpersonal touch to add a new layer to an old classic. Injecting cooperative interpersonal touch actions into Dance Dance Revolution's solo gameplay creates a new challenge wherein players must not just watch the screen, but their partners as well $(h-i i)$. These cooperative actions force players to win or lose as a team - players must work together in order to be successful. Finally, the aggressive use of interpersonal touch in Freqtric Robot Battle is an interesting way of acknowledging of the role of interpersonal touch in physical competition. Though slapping games are not for everyone, we believe that many male players would feel more comfortable slapping a male partner than they would holding their hand $(h-v)$. Such aggressive physical competition may even provide the basis for rewarding socialization - we are reminded of the schoolyard hand-slapping game known as "Red Hands", which, despite its painful consequences, is played in good fun and is often cause for laughter among its players $(h-i i i)$.

\subsection{Matchmaker}

Like "Get Lucky" Charms, Matchmaker [11] is a romantically-themed game. But whereas "Get Lucky" Charms puts its emphasis on overt, sexual romance, Matchmaker focuses on the "cute" aspects of love. In Matchmaker, interpersonal touch comes in the form of handholding - a gesture which is frequently used among couples to demonstrate affection and togetherness $(h-i v)$. Matchmaker is played directly on the surface of a touch-sensitive tabletop computer, with the players sitting side-by-side a configuration which easily allows players to hold hands with their partner $(h-v)$. Although the game is designed for couples, its relatively inoffensive use of touch means that it could also be enjoyed by other groups of players, such as parents and their children.

In Matchmaker, interpersonal touch serves two purposes. The first purpose is to promote an atmosphere of love and romance. In Matchmaker, handholding serves as a tangible symbol of the love that the players share. Matchmaker encourages players to touch as a way of showing their love for each other $(h-i i i)$. Interpersonal touch also serves as a form of cooperative interaction between players $(h-i)$. Handholding allows players to cure lovelorn Peeps so that they can be matched up again. Curing lovelorn Peeps is crucial part of Matchmaker's gameplay - not only does this mechanic provide much of Matchmaker's challenge $(h$-ii) but it also forces players to communicate and strategize in order to decide when they will hold hands, and when they will match up Peeps instead $(h$-iii).

\subsection{Prism Squad: GO!}

Although our current implementation of Prism Squad: GO! does not recognize interpersonal touch between its players, we are currently working to create an implementation of Prism Squad: GO! where players blend colors through touch rather than buttonpresses. Let us consider how these heuristics would apply to this implementation. 
Prism Squad: GO! is a team-based game which places significant emphasis on cooperation between players. In Prism Squad: GO!, this cooperation primarily manifests itself through the mechanic of "color-blending". Each player in Prism Squad: GO! embodies a particular color, and when two players touch, their colors combine. Interpersonal touch provides players with a simple and direct way to blend colors with their partners $(h-i)$. Color-blending (and by extension, interpersonal touch) is very important to Prism Squad: GO! - coordinating touch between three players at once provides the game with plenty of challenge $(h-i i)$ and serves as a source of ongoing strategic discussion between players $(h$-iii).

Prism Squad: GO! was designed for a broader audience than its predecessor Matchmaker, and so concerns over the social appropriateness of touch are much more valid here. Groups of friends who may otherwise enjoy gaming together might be turned off from Prism Squad because of its use of interpersonal touch $(h-i v)$. In our study of Prism Squad: GO!, several participants mentioned that Prism Squad would be an enjoyable party game, and we agree that parties are a likely niche for Prism Squad. The cooperative aspects of Prism Squad: GO! combined with its use of interpersonal touch could make Prism Squad an unique icebreaker (h-iii).

As a three-player game, Prism Squad presents a logistical challenge to working with interpersonal touch. It is very difficult to position three players in front of a screen in such a way that they can all see the screen, but also and can all touch each other at a moment's notice $(h-v)$. This logistical challenge could either be a frustrating obstacle, or a fun-but-frantic aspect of gameplay. We believe that the challenge of positing and repositioning your partners so that the appropriate people can touch at the appropriate times could provide a fun challenge in and of itself $(h-i i)$.

\section{Future Interpersonal Touch Interaction in Video Games}

In a previous section, we explored the current state of interpersonal touch-based video games. We have also presented a set of heuristics designed to guide the development of future interpersonal touch-based games. But what does the future of these games look like? How will touch-based interaction in games continue to evolve?

One possibility for future growth involves examining the role of touch-based games in the public social settings. Playtesters have often described games like Matchmaker and Prism Squad: GO! as "ice-breakers" - fun ways for new acquaintances to get to know each other. With this in mind, we believe it would be very interesting to see how players interact with interpersonal touch-based games in public social gatherings: parties, movie theatres, bars, or even speed-dating venues. Studying how players respond to the presence of these games in a public setting could produce very useful data about where (and to whom) interpersonal touch-based games are best suited.

Another avenue for future research is to explore the use of interpersonal touch in pervasive gaming. Interpersonal touch is a popular physical interaction technique - as such, it plays a key role in many physically-active games such as tag, hide-and-seek, and flag football. Traditionally, video games have lacked the mobility of these physically-active games. But with the popularization of mobile computing and wireless networking, a new class of computer-assisted pervasive video games are emerging, which allow players to play even as they live and move in the real world [5]. As the 
technology which supports pervasive gaming continues to improve, we believe it would be interesting to explore how interpersonal touch could mediate player-toplayer interaction in these pervasive games.

Finally, we believe there are opportunities for further growth in exploring the way in which touch is detected and processed. In existing touch-based games, interpersonal touch is treated as a binary phenomenon: either two players are touching, or they are not. Of course, this is a gross simplification of interpersonal touch: touch can be soft, or it can be forceful, it can be fleeting, or it can linger. Where and how two people touch can also change its meaning; slapping someone on the back is dramatically different from gently patting it. Due to the current state of interpersonal touchsensing technology, many of these nuances are lost to the game designer. A game like Matchmaker cannot distinguish a hug from a handshake [10]. Intimate Controllers uses touch-sensors to establish some sense of touch location, but it can only recognize touch on those sensors and nowhere else [2]. A game which could detect not only when, but where and how you touched your partner would have powerful implications for game design; imagine a video game where players must successfully complete a "secret handshake" in order to unleash a coordinated special attack. We believe that such a game would be a lighthearted but amusing way of promoting cooperation and interactivity through interpersonal touch.

\section{Conclusion}

Interpersonal touch-based games are a recent development in the ongoing evolution of physically-interactive games. Because these games are still so new, details on how to construct and evaluate effective touch-based-games are relatively unknown. In this paper, we have motivated interpersonal-touch based gameplay as a way of promoting inter-player interaction with the goal of supporting increased player socialization. We have also introduced four games which have taken advantage of this interaction technique in their gameplay mechanics: "Get Lucky" Charms, Freqtric Game, Matchmaker and Prism Squad: GO!

Based on the lessons we have learned from evaluating these projects, we have produced a set of five design heuristics for the effective use of interpersonal touch in video games. These heuristics are designed to expose the most important considerations for designing games based on interpersonal touch. Using these heuristics as a guide, we examined four existing touch-based games to show how our heuristics can be used as a framework for analyzing interpersonal touch-based games. Finally, we presented our thoughts on the future of interpersonal touch interaction in video games. We hope that this work will prove useful in the development of future games based on interpersonal touch.

\section{References}

1. Baba, T., Ushiama, T., Tsuruno, R., Tomimatsu, K.: Video Game that Uses Skin Contact as Controller Input. In: ACM SIGGRAPH 2007 Emerging Technologies (2007)

2. Burgoon, J.K., Walther, J.B., Baesler, E.J.: Interpretations, evaluations, and consequences of interpersonal touch. Human Communication Research 19, 237-263 (1992) 
3. Chowdhury, J.: Intimate Controllers (2007), http: / /www.jennylc.com/intimate_controllers

4. Crusco, A., Wetzel, C.: The Midas Touch: The Effects of Interpersonal Touch on Restaurant Tipping. Personality and Social Psychology Bulletin 10(4), 512-517 (1984)

5. Dreher, T.: Pervasive Games: Interfaces, Strategies and Moves (2008), http://iasl.uni-muenchen.de/links/NAPGe.html

6. Harlow, H.: The Nature of Love. American Psychologist 13, 673-685 (1958)

7. Koster, R.: A Theory of Fun for Game Design. Paraglyph Press, Scottsdale (2005)

8. Lazzaro, N.: Why We Play Games: Four Keys to More Emotion Without Story (2004), http: / / www. xeodesign.com/xeodesign_whyweplaygames.pdf

9. Voida, A., Greenberg, S.: Wii All Play: The Console Game as a Computational Meeting Place. In: Proceedings of CHI 2009, pp. 1559-1568. ACM, New York (2009)

10. Watts, C., Sharlin, E., Woytiuk, P.: Exploring Interpersonal Touch-Based Interaction and Player Socialization in Prism Squad: GO! In: 3rd Annual International Conference on Computer Games, Multimedia and Allied Technology, Singapore (2010)

11. Watts, C., Sharlin, E., Woytiuk, P.: Matchmaker: Interpersonal Touch in Gaming. In: Chang, M., Kuo, R., Kinshuk, Chen, G.-D., Hirose, M. (eds.) Learning by Playing. Gamebased Education System Design and Development. LNCS, vol. 5670, pp. 13-24. Springer, Heidelberg (2009) 